\title{
Out of sequence
}

\section{Synthesis, sequencing and other DNA-related matters}

StratoSpheres Plugs

Polymer Laboratories www.polymerlabs.com Resin to be cheerful

StratoSpheres Plugs are resin-loaded devices designed to simplify the synthesis of small molecules and peptides. The resin is entrapped so that it reacts in a normal manner, but contained in a pre-formatted, pre-packaged format eliminating the need to weigh loose resin. The composite plugs are made by co-sintering HDPE material with solid-phase synthesis resins, solid supported reagents or scavengers. StratoSpheres Plugs suit most 48or 96-well formats and can be used in all types of agitation systems, including magnetic stirring or vortexing or with automation devices.

\section{0/3730xI DNA analysers}

Applied Biosystems www.applera.com Increase productivity twofold

Applied Biosystems' 3730 and 3730xl DNA analysers are marketed as next generation production-scale DNA analysis systems. The 3730 analyser, a 48-capillary electrophoresis system, combines technology from the established ABI PRISM3100 genetic analyser with the latest in automation and optics, to meet the needs of high throughput. The 3730 platform can be upgraded to $3730 \mathrm{xl}$ specification, the 96-capillary successor to the PRISM 3700. Researchers and production facilities are expected to use the 3730xl DNA analyser for production sequencing and the 3730 for a wider range of sequencing and fragment analysis applications.

\section{Universal ISH Detection Kits}

Innogenex www.innogenex.com Search and stain

The Universal ISH (in situ hybridization) Kit offers a complete non-radioactive system for localization of DNA and RNA sequences in all cells and tissues, including rodent species. The ready-to-use kit utilizes proprietary signal amplification technology for the colorimetric detection of fluorescein and digoxin

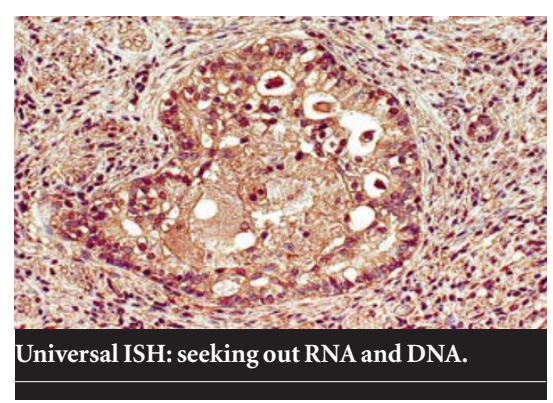

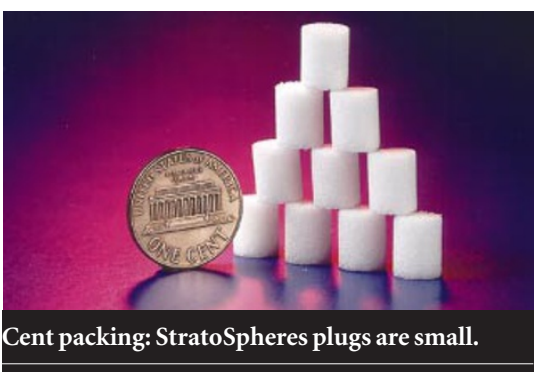

labelled probes. Serial amplification is used for high sensitivity, and detection reagents and protocols are optimized to maximize signal-to-noise ratios. The kits are available in two formats for detection of fluorescein labelled probes and digoxin.

\section{In-Fusion PCR Cloning System}

BD Biosciences www.bdbiosciences.com Rapid high-throughput PCR

The BD In-Fusion PCR Cloning System enables directional and efficient highthroughput cloning of PCR fragments. This method is compatible with any thermostable polymerase and does not require blunt end polishing or the presence of A-overhangs. InFusion System is claimed to generate precise, directional constructs that are ready for expression analysis with BD Biosciences' Creator Gene Cloning and Expression System.

\section{Cloned AMV First-Strand Synthesis}

Invitrogen Corporation www.invitrogen.com Go into reverse transcription

Cloned AMV First-Strand Synthesis Kit combines the technology of Invitrogen's Cloned AMV RT with the convenience of optimized, pre-qualified reagents. Everything necessary for high-quality, routine reverse transcription is provided, including highly purified Cloned AMV RT.

\section{All-Gone}

Amresco

www.amresco-inc.com Prevent nuclease contamination

The All-Gone Glass Plate Kit offers a quick, convenient and efficient way to safeguard experiments from RNase and DNase contamination. It is a three-component kit that can eliminate nuclease contamination associated with preparing glass plates for electrophoresis experiments. NucleasEliminator Wipes are used to remove nuclease contamination from the glass plate surface. The rinse solution is for rinsing the plate off, and Acryl-Glide Wipes prevent the glass plates from sticking to acrylamide gels. The All-Gone kit contains sufficient materials to prepare 10 slab gel sequencing plate sets or 20-40 mini gel plate sets and can be stored at room temperature.

Biochrom 30

Biochrom www.biochrom.co.uk Manifold improvements

Hardware improvements incorporated in the Biochrom 30 amino acid analyser include new pumps with ceramic heads for dependable high-pressure operation and future expandability, and a new manifold-based valving system that improves reliability by reducing the potential for leaks. The Biochrom 30 software fully integrates instrument control and data handling functions. Coupled with a new Graphical User Interface, this is claimed to make operation intuitive and easy, allowing flexible data export and customized reporting.

\section{Westran Clear Signal/ S PVDF}

\section{Schleicher \& Schuell BioScience}

www.arraying.com

Branching out

Schleicher \& Schuell's PVDF membrane is optimized for proteomics, western blotting and protein sequencing applications. The previous single Westran membrane has been replaced with two new formulations, Westran Clear Signal and Westran S. With uniform pore structures, Westran Clear Signal is a 0.45 $\mu \mathrm{m}$ pore membrane designed to produce low background and strong signal after western blotting. Westran S has increased chemical resistance, high tensile strength and a $0.2 \mu \mathrm{m}$ pore size, making it ideal for protein sequencing applications. Westran Clear Signal and Westran S PVDF may be ordered directly from S\&S BioScience by calling 1-800-245-4024.

These notes are compiled in the Nature office from information provided by the manufacturers. 